\title{
Magnesium reduces $N$-methyl-D-aspartate (NMDA)-mediated brain injury in perinatal rats
}

\author{
John W. McDonald', Faye S. Silverstein ${ }^{2}$ and Michael V. Johnston ${ }^{3}$ \\ 'Neuroscience and Medical Scientist Training Program, 'Departments of Pediatrics and Neurology. \\ University of Michigan Medical School, Ann Arbor, MI (U.S.A.) and ' Departments of Neurolog.' and \\ Pediatrics, The Johns Hopkins Universify of Medicine and the Kennedy Institute, Baltimore, MD /U.S.A.)
}

(Received 25 July 1989; Revised version received 3 October 1989; Accepted 4 October 1989)

Key words: Magnesium; $N$-Methyl-D-aspartate; Neurotoxicity; Neuroprotection; Rat brain; Perinatal

We evaluated the neuroprotective effects of systemically administered magnesium against $\mathrm{N}$-methyl-Daspartate (NMDA)-mediated brain injury in perinatal rats. Postnatal day (PND) 7 rats received unilateral intrastriatal injections of 25 nmol NMDA followed 15 min later by single or multiple doses of magnesium intraperitoneally (i.p.). Animals were sacrificed five days later and the severity of brain injury was assessed by comparison of the weights of the injected and contralateral cerebral hemispheres. NMDA injection reduced the weight of the injected cerebral hemisphere by $31 \pm 3 \%$. Single doses of magnesium reduced the severity of NMDA-induced brain injury in a dose-dependent fashion $(2 \mathrm{mmol} / \mathrm{kg}, 29 \pm 11 \%$ protection: $3 \mathrm{mmol} / \mathrm{kg}, 52 \pm 12 \%$ protection; $4 \mathrm{mmol} / \mathrm{kg}, 62 \pm 7 \%$ protection). Multiple doses of magnesium reduced brain injury by $65 \pm 4 \%$. These data demonstrate that systemically administered magnesium antagonizes the neurotoxic effects of NMDA in vivo in perinatal rats.

The $N$-methyl-D-aspartate (NMDA)-type excitatory amino acid (EAA) receptor is coupled to a $\mathrm{Ca}^{2+}$ permeable cationic channel; the receptor/channel complex includes several regulatory sites $[2,24]$. Physiological concentrations of magnesium reduce responses to NMDA by blocking the NMDA-associated ionophore in a voltage-dependent manner [2]. Intrastriatal injection of NMDA typically produces focal brain injury in PND 7 rats $[17,19,20]$. Competitive and non-competitive NMDA receptor antagonists attenuate this damage in PND 7 rats in a dose-dependent fashion $[20,21]$. Furthermore, these antagonists also limit NMDA neurotoxicity in adult rats and in neocortical cultures [11, 12]. However, few of these antagonists are approved for use in humans. Although its mechanism of action is unknown, magnesium has been used therapeutically for many years in obstetrics for the treatment of eclampsia [4]. This study examines the neuroprotective effects of magnesium against NMDA-mediated brain injury in PND 7 rats.

Correspondence: M.V. Johnston, Kennedy Institute, Room 506, 707 N. Broadway, Baltimore, MD 21205. U.S.A. 
PND 7 Sprague-Dawley albino rats, anesthetized with ether, received unilateral intrastriatal stereotaxic injections of $25 \mathrm{nmol}$ NMDA $(0.5 \mu$ l injection vol., $\mathrm{pH} 7.4)$ using a Hamilton syringe (26 gauge) as detailed previously [18, 19, 22]. With bregma as a reference, injection coordinates were AP $2.0 \mathrm{~mm}, \mathrm{ML} 2.5 \mathrm{~mm}$, at a depth of $4 \mathrm{~mm}$ from dura. Magnesium (sulfate) was dissolved in isotonic saline ( $\mathrm{pH} 7.4$ ) and administered by i.p. injection in single or multiple doses (injection vol. $=0.05 \mathrm{ml}$ ) beginning $15 \mathrm{~min}$ after the NMDA injection. Two magnesium treatment protocols were tested; (1) a single dose ( $2 \mathrm{mmol} / \mathrm{kg}, n=4 ; 3 \mathrm{mmol} / \mathrm{kg}, n=4 ; 4 \mathrm{mmol} / \mathrm{kg}, n=4$;) and (2) 6 doses (each $1.8 \mathrm{mmol} / \mathrm{kg}$ given at variable time points over a $6 \mathrm{~h}$ period, total dose of $11 \mathrm{mmol} / \mathrm{kg})$ as necessary to control behavioral signs of seizures $(n=8)$. Isotonic saline-treated NMDA-injected controls were included in each protocol following the appropriate dosage schedule (protocol 1, $n=4$ ); protocol 2, $n=8$ ). A 3rd group of NMDA-injected rats $(n=8)$ received equimolar $\mathrm{NaCl}$ injections in vehicle following the dosage schedule of protocol 2 (six $1.8 \mathrm{mmol} / \mathrm{kg}$ injections). Additional, non-NMDA-injected rats were given i.p. injections $(2 \mathrm{mmol} / \mathrm{kg})$ of either magnesium $(n=6)$ or $\mathrm{NaCl}(n=6)$. Animals' body temperatures were maintained at normothermia $\left(35.5 \pm 2^{\circ} \mathrm{C}\right.$ surface temperature) for $2 \mathrm{~h}$ following treatment using warming lamps and electronic surface probes. All animals were decapitated on PND 12, five days after NMDA injection. The degree of resulting brain injury was quantitated by comparison of the weights of the injected $(I)$ and contralateral $(C)$ cerebral hemisphere weights using the formula, $\%$ Damage $=100(\mathrm{C}-I) / C$, expressed as mean\pm S.E.M. [18, 22]. This is a sensitive and reliable method for quantitating unilateral brain injury in this experimental model; \%Damage values are highly correlated $\left(r^{2}=0.99, P<0.001\right)$ with ipsilateral reductions in both choline acetyl transferase activity and regional cross-sectional areas [18, 20, 22]. To assess neuroprotective efficacy $\%$ Protection values were calculated as: $\%$ Protection $=100 *[1-(\%$ Damage magnesium-treated/\%Damage saline-treated)]. Statistical comparisons were made using one-way ANOVA except where otherwise noted. Saline-treated NMDA-injected controls were pooled for statistical comparisons since no significant differences were observed between groups ( $P=$ N.S., independent $t$-test).

Intrastriatal injection of NMDA (25 nmol) produced tonic-clonic seizure activity and in animals sacrificed five days later resulted in predominantly unilateral confluent brain necrosis involving the corpus striatum and extending into the thalamus, dorsal hippocampus, and adjacent neocortex [17, 19]. In saline-treated groups, NMDA (25 $\mathrm{nmol})$ injections reduced the weight of the injected hemisphere by $31.3 \pm 2.5 \%$ (mean \pm S.E.M., $n=10$ ) relative to the contralateral cerebral hemisphere and resulted in $17 \%$ mortality rate. Administration (i.p.) of magnesium, but not $\mathrm{NaCl}$, reduced the behavioral signs of seizures and decreased muscle tone. The highest dose of magnesium ( $4 \mathrm{mmol} / \mathrm{kg}$ ) produced short periods $(10-30 \mathrm{~s})$ of apnea. Higher doses resulted in prolonged apnea and increased the mortality rate. Single doses of magnesium significantly reduced the extent of NMDA-mediated brain injury in a dose-dependent manner $(P<0.001$, ANOVA, Fig. 1). Multiple doses of magnesium reduced the extent of brain injury by $65 \pm 4 \%$ relative to saline treated, NMDA-injected controls ( $P<0.001$, ANOVA). In contrast, multiple doses of equi- 


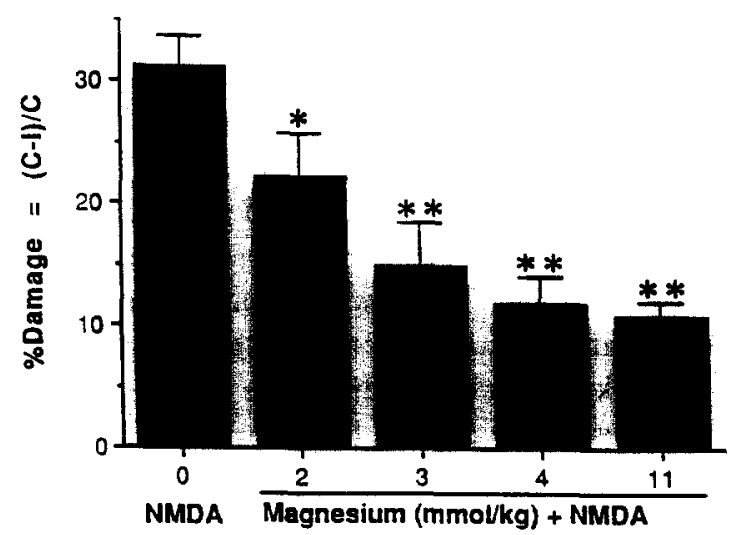

Fig. 1. Quantitative neuroprotective effects of single and multiple doses of magnesium against NMDAmediated brain injury. Unilateral intrastriatal stereotaxic injections of NMDA $(25 \mathrm{nmol})$ were performed in ether anesthetized PND 7 rats. Fifteen min later, single $(2,3,4 \mathrm{mmol} / \mathrm{kg}, n=4 / \mathrm{dose})$ or multiple (6 injections of $1.8 \mathrm{mmol} / \mathrm{kg}$ each over $6 \mathrm{~h}, n=8)$ doses of magnesium were administered i.p. in $0.05 \mathrm{ml}$ saline. NMDA-injected littermate controls received equivalent volumes of saline $(n=10)$. Animals were decapitated 5 days later on PND 12. The severity of resulting brain injury was assessed by comparison of the weights of the injected $(I)$ and contralateral $(C)$ cerebral hemisphere weights and expressed as \%Damage (mean \pm S.E.M.) using the formula on the vertical axis. ${ }^{*} P<0.05, " P<0.001$. ANOVA. magnesiumtreated vs saline treated.

molar $\mathrm{NaCl}$ increased NMDA-induced brain injury by $25 \pm 7 \%(P<0.001$, independent $t$-test; $39.2 \pm 2.2 \%$ Damage, $\mathrm{NaCl}, n=8$ vs $31.3 \pm 2.5 \%$ Damage, isotonic saline treated controls, $n=10$. The mortality rate was $0 \%$ in groups treated with magnesium or $\mathrm{NaCl}$.

Both competitive and non-competitive NMDA receptor antagonists attenuate NMDA-mediated brain injury [11, 19-21]. These data demonstrate that magnesium can also limit NMDA-induced brain injury. In the same animal model, complete neuroprotection against NMDA toxicity is possible with MK-801 and TCP, two non-competitive NMDA antagonists, and with CGS-19755, a competitive NMDA antagonist. The level of neuroprotection achieved by magnesium against NMDA toxicity in this experimental model is equivalent to the maximal degrees of protection produced by the non-competitive NMDA antagonists, phencyclidine (PCP), ketamine and dextromethorphan, the competitive NMDA antagonist CPP, and the glycine modulatory site antagonist HA-966 in this experimental model [18, 20-22]. It is possible that combined treatment with magnesium and other NMDA receptor antagonists may potentiate their neuroprotective effects.

Several mechanisms could account for magnesium's neuroprotective properties [ 1 , 8]. High concentrations of magnesium may directly block the NMDA-associated ionophore [2]. Furthermore, magnesium acts as a $\mathrm{Ca}^{2+}$ antagonist and can inhibit neurotransmitter release and reduce seizure activity at high concentrations $[3,14]$. Our study does not directly address the mechanisms of magnesium's neuroprotective 
effects. However, several lines of evidence suggest that neither inhibition of neurotransmitter release nor anticonvulsant actions play a major role in magnesium's neuroprotective effects in this experimental model. The dosages of magnesium used in this study did not block neuromuscular transmission. In addition, in this experimental model adenosine, an inhibitor of synaptic neurotransmitter release, does not reduce NMDA toxicity [18]. Nor is it likely that magnesium's neuroprotective effects can be attributed to anticonvulsant properties since anticonvulsants (e.g. pentobarbital, carbamazepine) which reduce the behavioral signs of seizures are not neuroprotective [18]. Further studies are necessary to determine if the protective effects of magnesium are specific for NMDA-mediated damage or whether magnesium can also reduce other forms of excitotoxic brain injury. The mechanism by which multiple doses of hyperosmolar $\mathrm{NaCl}$ enhance NMDA-mediated brain injury are not clear but may be related to intravascular hypernatremia and hypovolemia.

Excessive activation of NMDA receptors has been implicated in the pathogenesis of neuronal injury in a number of acute neurologic disorders including hypoxiaischemia, physical brain trauma, and prolonged seizures $[5,9,15,16,25]$. The finding that magnesium reduced NMDA-mediated brain injury supports in vitro and in vivo studies which indicate that magnesium can limit neuronal injury resulting from hypoxia and ischemia and traumatic brain injury [5, 9, 13, 16, 23, 25, 26]. Magnesium normally does not readily penetrate the fully developed blood-brain barrier (BBB), but $\mathrm{BBB}$ permeability is greater in infants and during seizures, hypoxia-ischemia and other acute neurologic disorders in adults $[4,7,10]$. Thus, magnesium may provide effective therapy in these conditions when administered systemically. The experimental evidence of magnesium's neuroprotective properties in the developing and adult brain, together with its ability to penetrate the BBB under certain conditions, its proven clinical utility in obstetrics, and the absence of neurotoxicity suggest that this agent could provide an important therapeutic approach to limit excitotoxic brain injury in humans $[3,4,9,10,13,23,25,26]$.

Supported by USPHS Grants 1P01NS19613 (M.V.J.) and NS 26142 (F.S.S.). J.W.M. is a recipient of a MSTP fellowship (5 T32 6M07863-07).

1 Aikawa, J.K., Magnesium: Its Biological Significance, CRC Press, Boca Raton, F1, 1981, pp. 21-29.

2 Ascher, P. and Nowak, L., Electrophysiological studies of NMDA receptors, Trends Neurosci., 10 (1987) $284-288$.

3 Borges, L.F. and Grucer, G., Effect of magnesium on epileptic foci, Epilepsia, 19 (1978) 81-91.

4 Chesley, L.C., Hypertensive disorders in pregnancy, Appleton-Century-Crofts, New york, 1978.

5 Clark, G.D. and Rothman, S.M., Blockade of excitatory amino acid receptors protects anoxic hippocampal slices, Neuroscience, 21 (1987) 665-671.

6 Cortez, S.C., McIntosh, T.K. and Noble, L.J., Experimental fluid-percussion brain injury: vascular disruption and neuronal and glial alterations, Brain Res., 482 (1989) 271-282.

7 Cutler, R.W.P., Lorenzo, A.V. and Barlow, C.F., Changes in blood-brain barrier permeability during pharmacologically induced convulsions, Brain Res., 29 (1968) 367-384.

8 Ebel, H. and Gunther, T., Magnesium metabolism: a review, J. Clin. Chem. Clin. Biochem., 18 (1980) 257-270

9 Fadden, A.I. and Simon, R.P., A potential role for excitotoxins in the pathophysiology of spinal cord injury, Ann. Neurol., 23 (1988) 623-626. 
10 Fishman, R.A., Neurological aspects of magnesium metabolism, Arch. Neurol., 12 (1965) 562-569.

11 Foster, A.C., Gill, R., Kemp, J.A. and Woodruff, G.N., Systemic administration of MK-801 protects against ischemia-induced hippocampal neurodegeneration in the gerbil, J. Neurosci., 7 (1987) 33433349

12 Goldberg, M.P.. Viseskul, V. and Choi, D.W., Phencyclidine receptor ligands attenuate cortical neuronal injury after $N$-methyl-D-aspartate exposure or hypoxia, J. Pharmacol. Exp. Ther., 245 (1988) $1081-1087$.

13 Kass, I.S., Cottrell, J.E., and Chambers, G., Magnesium and cobalt, not nimodipine, protect neurons against anoxic damage in the rat hippocampal slice, Anesthesiology, 69 (1988) 710-715.

14 Katz. B., Nerve, Muscle, and Synapse, McGraw-Hill, New York, 1989, pp. 129-141.

15 Labuyere, J., Fuller, T.A., Olney, J.W., Price, M.T., Zormuski, C. and Clifford, D., Phencyclidine and ketamine protect against kainic acid-induced seizure related damage, Soc. Neurosci. Abstr., 12 (1986) 344.

16 McDonald, J.W., Silverstein, F.S. and Johnston, M.V., MK-801 protects the neonatal brain from hypoxic-ischemic damage, Eur. J. Pharmacol., 140 (1987) 359-361.

17 McDonald, J.W., Silverstein, F.S. and Johnston, M.V., Neurotoxicity of $N$-methyl-D-aspartate is markedly enhanced in developing rat central nervous system, Brain Res., 459 (1988) 200-203.

18 McDonald, J.W., Silverstein. F.S. and Johnston, M.V., Comparison of neuroprotective effects of competitive and non-comppetitive NMDA antagonists against NMDA mediated neurotoxicity in an in vivo perinatal rat model. In E.A. Cavalheiro, J. Lehmann, L. Turski (Eds.), Recent Advances in Excitatory Amino Acid Research, Vol. 46, Liss, New York, 1988, pp. 601-604.

19 McDonald, J.W., Silverstein. F.S., Cardona, D., Hudson, C., Chen, R. and Johnston, M.V., Systemic administration of MK-801 protects against $N$-methyl-D-aspartate and quisqualate mediated neurotoxicity in perinatal rats, Neurosci., in press.

20 MCDonald, J.W., Silverstein, F.S. and Johnston, M.V., Neuroprotective effects of MK-801, TCP. $\mathrm{PCP}$, and CPP against $N$-methyl-D-aspartate induced neurotoxicity in an in vivo perinatal rat model, Brain Res., 490 (1989) 33-40.

21 McDonald, J.W., Uckele, J., Silverstein, F.S. and Johnston, M.V., HA-966 (1-Hydroxy-3-aminopyrrolidone-2) selectively reduces $N$-methyl-D-aspartate (NMDA)-mediated brain damage, Neurosci. Lett. . $104(1989) 167-170$.

22 McDonald, J.W., Roeser, N.F., Silverstein. F.S. and Johnston, M.V., Quantitative assessment of neuroprotection against NMDA-mediated brain injury, Exp. Neurol., in press.

23 McIntosh, T.K., Vink, R., Yamakami, I. and Faden, A.I., Magnesium protects against neurological deficit after brain injury, Brain Res., 482 (1989) 252-260.

24 Reynolds. I.J. and Miller, R.J., [3H]MK-80I binding to the NMDA receptor/ionophore complex is regulated by divalent cations: evidence for multiple regulatory sites, Eur. J. Pharmacol., 151 (1988) 103.112.

25 Rothman, S.. Synaptic release of excitatory amino acid neurotransmitter mediates anoxic neuronal death, J. Neurosci., 4 (1984) 1884-1891.

26 Vacanti, F.X. and Ames, A., Mild hypothermia and $\mathrm{Mg}^{++}$protect against irreversible damage during CNS ischemia, Stroke, 15(1984) 695-698. 